\title{
Analytic calculation of field-strength correlators
}

\author{
Yu.A.Simonov \\ State Research Center \\ Institute of Theoretical and Experimental Physics, \\ Moscow, 117218 Russia
}

\begin{abstract}
Field correlators are expressed using background field formalism through the gluelump Green's functions. The latter are obtained in the path integral and Hamiltonian formalism. As a result behaviour of field correlators is obtained at small and large distances both for perturbative and nonperturbative parts. The latter decay exponentially at large distances and are finite at $x=0$, in agreement with OPE and lattice data.
\end{abstract}

\section{Introduction}

The Method of Field Correlators (MFC), suggested some years ago [1] (see [2] for a review) has successfully produced a large number of results in nonperturbative QCD, in particular predicting hadron masses in good agreement with lattice and experimental data (see e.g. 3 for a review) and unambigiously explaining linear confinement [4], [5].

In doing so MFC is exploiting the field correlators known from lattice data [6] as input parameters. Recent discovery of Casimir scaling for static potentials [7, 8] allows to neglect all correlators except for the lowest ones (bilocal or Gaussian) with accuracy about $1 \%$ and this fact reduces input parameters essentially to the string tension $\sigma$ for the bulk of the hadron spectrum and in addition correlation length $T_{g}$ for hadron spin splittings. Thus all spin-averaged spectrum of hadrons, namely light mesons [9], heavy 
quarkonia [10], hybrids [3, 11, 12], heavy-light mesons [13], glueballs [14] and baryons [15] are calculated through the only parameter $\sigma$ in good agreement $(\sim 10 \%)$ with experimental and lattice data.

Similar situation occurs for the background perturbation theory in the real QCD vacuum, where the only additional parameter $m_{B}$, calculated in terms of $\sigma$, can be used to construct a new improved perturbation series without Landau ghost pole and IR renormalons [16. With all that the situation in MFC is not yet satisfactory, since till now field correlators have not been computed analytically within the method itself. The first attempts in this direction have been done in [17] where equations for field correlators have been first written and the correlation length $T_{g}$ was computed in terms of $\sigma$, in reasonable agreement with lattice data [6]. (For earlier developments in the framework of stochastic quantization method see [18. Resulting equations there however contain path integrals and are too complicated for practical use).

Recently new objects - gluelumps - have been introduced [19], which represent bound states of a valence gluon in the field of static gluonic charge an adjoint equivalent of heavy-light mesons. Spectrum of gluelumps was calculated on the lattice [20] and analytically in [21], being in general agreement with each other.

It was realized subsequently, that gluelump Green's functions and field correlators are the same, if in - and out - states of gluelumps are constructed with the help of field strength operators.

For the analytic calculations in 21] the Background Field Formalism (BFF) was used 22, 16, which allows to construct gluelump states and the Hamiltonian in a very simple local form. Essentially the valence gluon operators have been introduced in [21] and used to construct the whole hierarchy of gluelump states, which agrees resultatively with the states used in lattice calculations 20]. Therefore one can use now the simple technic of [21] to calculate the gluelump Green's functions, and through them, the field correlators. In this way the logic of the method is closed, since in principle one can calculate all field correlators and through them all physical quantities in terms of only one input parameter - the string tension. In practice calculations are approximate and use Gaussian approximation, when only lowest correlators are retained with later improvements.

The full implementation of this program requires solution of three basic problems: 
1. Dynamical definitions of perturbative and nonperturbative. In terms of $\mathrm{BFF}$ one needs to understand how the nonperturbative fields are constructed in contrast to the perturbative valence gluon fields.

2. Gaussian dominance, i.e. suppression of contributions of quartic and higher field correlators.

3. Explicit relation between $\Lambda_{Q C D}$ and $\sigma$, so that all resulting quantities containing both perturbative and nonperturbative contributions, can be expressed through only one scale parameter.

In the present paper only partial answers will be given to the questions 1 and 3, nevertheless allowing to pave the road for future developments.

The paper is organized as follows. In section 2 the field correlators are written in the framework of BFF and the correlator $D_{1}$ is expressed in terms of gluelump Green's functions. In section 3 a similar detailed study of the confining correlators $D(x)$ is done and its behaviour is defined at small and large $x$. In section 4 properties and selfconsistency of field correlators are discussed. Five appendices contain details of derivation of basic equations in the main text.

\section{Field correlators in the Background For- malism}

We start with the standard definitions of the field correlator functions $D(x)$ and $D_{1}(x)$, defined as in [1]

$$
\begin{gathered}
\frac{g^{2}}{N_{c}}\left\langle\operatorname{tr}_{f}\left(F_{\mu \nu}(x) \Phi(x, y) F_{\lambda \sigma}(y) \Phi(y, x)\right)\right\rangle \equiv D_{\mu \nu, \lambda \sigma}(x, y)= \\
=\left(\delta_{\mu \lambda} \delta_{\nu \sigma}-\delta_{\mu \sigma} \delta_{\nu \lambda}\right) D(x-y)+\frac{1}{2}\left(\frac{\partial}{\partial x_{\mu}} h_{\lambda} \delta_{\nu \sigma}+\text { perm. }\right) D_{1}(x-y)
\end{gathered}
$$

where $h_{\mu}=x_{\mu}-y_{\mu}, \quad \Phi(x, y)=P \exp i g \int_{y}^{x} A_{\mu} d z_{\mu}$ and $t r_{f}$ is the trace in the fundamental representation. Our final aim in this section will be to connect $D(x), D_{1}(x)$ to the gluelump Green's functions. If the latter are defined as it is done on the lattice [19, 20, i.e. with in- and out- states constructed with the help of field strength operators, then the connection is trivial

$$
D_{\mu \nu, \lambda \sigma}(x, y)=\frac{g^{2}}{2 N_{c}^{2}}\left\langle\operatorname{tr}_{a} \hat{F}_{\mu \nu}(x) \hat{\Phi}(x, y) \hat{F}_{\lambda \sigma}(y)\right\rangle
$$


and here all dashed letters stand for operators in the adjoint representation. These Green's functions are however not accessible for analytic calculation and to proceed one needs to use BFF [16], where the notions of valence gluon field $a_{\mu}$ and background field $B_{\mu}$ are introduced, so that total gluonic field $A_{\mu}$ is written as

$$
A_{\mu}=B_{\mu}+a_{\mu}
$$

As it can be shown with the help of the 'tHooft identity [16], the independent functional integration over $D B D a$ does not contain the double counting, and one can proceed to define perturbation series in powers of $\left(g a_{\mu}\right)$ as it was done in [16. However for our purposes here we need a more explicit definition of the background and of field separation in (31). To this end one can use the main idea suggested in [17] which we explain here in the most simple form. Namely, let us single out some color index $a$ and fix it at a given number. The field $A_{\mu}^{a}$ will be identified with $a_{\mu}^{a}$ while the rest of fields will be called $A_{\mu}^{b}=B_{\mu}^{b}, \quad b \neq a$. In the averaging over fields $B_{\mu}^{b}$, and only after this done one integrates also fields $a_{\mu}^{a}$ The essential point is as was shown in [17] that the integration over $D B_{\mu}^{b}$ will provide a white adjoint string for the gluon $a_{\mu}^{a}$, which keeps the color index a unchanged. This is the basic physical mechanism behind this background technic and it is connected to the properties of ensemble gluons: 1) even for $N_{c}=3$ one has one field $a_{\mu}^{a}$ and 7 fields $B_{\mu}^{b} 2$ ) confining string is a colorless object and therefore the singled out color index $a$ can be preserved during interaction process of the valence gluon $a_{\mu}^{a}$ with the rest of gluons $\left(B_{\mu}^{b}\right)$. These remarks make explicit the notions of the valence gluon and background field and will be used in what follows.

Using (3) one can write the total field operator $F_{\mu \nu}(x)$ as follows

$$
\begin{gathered}
F_{\mu \nu}(x)=\partial_{\mu} A_{\nu}-\partial_{\nu} A_{\mu}-i g\left[A_{\mu}, A_{\nu}\right]= \\
=\partial_{\mu}\left(a_{\nu}+B_{\nu}\right)-\partial_{\nu}\left(a_{\mu}+B_{\mu}\right)-i g\left[a_{\mu}+B_{\mu}, a_{\nu}+B_{\nu}\right]= \\
=\hat{D}_{\mu} a_{\nu}-\hat{D}_{\nu} a_{\mu}-i g\left[a_{\mu}, a_{\nu}\right]+F_{\mu \nu}^{(B)} .
\end{gathered}
$$

Here the term, $F_{\mu \nu}^{(B)}$ contains only the field $B_{\mu}^{b}$. It is clear that when one averages over field $a_{\mu}^{a}$ and sums finally over all color indices $a$, one actually exploits all the fields with color indices from $F_{\mu \nu}^{(B)}$, so that the term $F_{\mu \nu}^{(B)}$ can be omitted, if summing over all $a$ is presumed to be done at the end of calculation. In this section we shall concentrate on the first two terms on the r.h.s. of (4I), leaving discussion of the term $\left[a_{\mu}, a_{\nu}\right]$ to the section 3 . 
Assuming the background Feynman gauge, $D_{\mu} a_{\mu}=0$, 21] we shall define now the gluelump Green's function as

$$
G_{\mu \nu}(x, y)=\left\langle\operatorname{tr}_{a} a_{\mu}(x) \hat{\Phi}(x, y) a_{\nu}(y)\right\rangle
$$

and the function $D_{\mu \nu, \lambda \sigma}$ can be written as

$$
D_{\mu \nu, \lambda \sigma}(x, y)=D_{\mu \nu, \lambda \sigma}^{(0)}+D_{\mu \nu, \lambda \sigma}^{(1)}+D_{\mu \nu, \lambda \sigma}^{(2)}
$$

where the superscript $0,1,2$ denotes the power of $g$ coming from the term $(i g)\left[a_{\mu}, a_{\nu}\right]$ in (4)

For the following one can use the relation

$$
\begin{gathered}
\left\langle\operatorname{tr}_{a}\left(D_{\mu} a_{\nu}(x)\right) \hat{\Phi} \chi\right\rangle=\frac{\partial}{\partial x_{\mu}}\left\langle\operatorname{tr}_{a} a_{\nu} \hat{\Phi} \chi\right\rangle- \\
-\left\langle\operatorname{tr}_{a} a_{\nu}(x) \delta_{\mu}(x) \hat{\Phi} \chi\right\rangle
\end{gathered}
$$

where the following notation is used for the contour differentiation (see [23] for details and earlier refs.)

$$
\delta_{\mu}(x) \hat{\Phi}(x, y)=i g \int_{y}^{x} d z_{\lambda} \alpha(z, y) \hat{\Phi}(x, z) \hat{F}_{\mu \lambda}(z) \hat{\Phi}(z, y) .
$$

Analogously for differentiation in the end point one has

$$
\hat{\Phi}(x, y) \overleftarrow{\delta_{\mu}}(y)=-i g \int_{y}^{x} d z_{\lambda} \alpha(z, x) \hat{\Phi}(x, z) \hat{F}_{\mu \lambda}(z) \hat{\Phi}(z, y)
$$

where $\alpha(z, y)=\left|\frac{z-y}{x-y}\right|, \quad \alpha(z, x)=\left|\frac{x-z}{x-y}\right|$.

One has

$$
\begin{gathered}
D_{\mu \nu, \lambda \sigma}^{(0)}(x, y)=\frac{g^{2}}{2 N_{c}^{2}}\left\{\frac{\partial}{\partial x_{\mu}} \frac{\partial}{\partial y_{\lambda}}\left\langle t r_{a} a_{\nu}(x) \hat{\Phi}(x, y) a_{\sigma}(y)\right\rangle+\right. \\
+ \text { perm. }-\frac{\partial}{\partial y_{\lambda}}\left\langle\operatorname{tr}_{a} a_{\nu}(x) \delta_{\mu}(x) \hat{\Phi} a_{\sigma}(y)\right\rangle-\text { perm. } \\
-\frac{\partial}{\partial x_{\mu}}\left\langle t r_{a} a_{\nu}(x) \hat{\Phi}(x, y) \overleftarrow{\delta_{\lambda}}(y) a_{\sigma}(y)\right\rangle+ \\
\left.+\left\langle\operatorname{tr}_{a} a_{\nu}(x) \delta_{\mu}(x) \hat{\Phi}(x, y) \overleftarrow{\delta_{\lambda}}(y) a_{\sigma}(y)\right\rangle+\text { perm }\right\}
\end{gathered}
$$


From the structure of the r.h.s. of (10) it is clear that all terms except the last one (and its permutations) contribute to the function $D_{1}$, which has the form of the full derivative, cf. Eq.(11). In what follows it is convenient to take spacial indices for $\mu, \nu$ in (5) $, \mu \rightarrow i, \nu \rightarrow k$, and consider the correlator of color-electric fields $D_{4 i, 4 k}(x, y)$, where $x, y$ are taken on the time axis. Hence the integral in (8), (9) is over $d z_{4}$ and for $\mu=4$ both terms (8), (9) disappear. We can also write for the gluelump Green's function $G_{\mu \nu}(x, y)=\delta_{\mu \nu} f\left((x-y)^{2}\right)$.

As a result one obtains from (10) the following connection of $D^{(0)}$ and $f\left((x-y)^{2}\right)$

$$
D_{4 i, 4 k}^{(0)}(x, y)=\frac{g^{2}}{2 N_{c}^{2}}\left\{\frac{\partial}{\partial x_{4}} \frac{\partial}{\partial y_{4}} \delta_{i k} f\left((x-y)^{2}\right)+\frac{\partial}{\partial x_{i}} \frac{\partial}{\partial y_{k}} f\left((x-y)^{2}\right)\right\}
$$

on the other hand using (1) with $h_{\mu} \equiv x_{\mu}-y_{\mu}$ one can express $D^{(0)}$ through $D_{1}$ as

$$
D_{4 i, 4 k}(h)=\delta_{i k} D(h)+\frac{1}{2}\left(\frac{\partial}{\partial x_{4}} h_{4} D_{1} \delta_{i k}+\frac{\partial}{\partial x_{i}} h_{k} D_{1}\right)
$$

and for $h_{i}=0, i=1,2,3, h_{4} \neq 0$ one obtains

$$
D_{1}(x)=-\frac{2 g^{2}}{N_{c}^{2}} \frac{d f\left(x^{2}\right)}{d x^{2}}
$$

To obtain information about the gluelump Green's function $G_{\mu \nu}$ one can use the path-integral representation of $G_{\mu \nu}(x, y)$ in the Fock-FeynmanSchwinger (FFS) formalism (see [24] for reviews and original references), which was exploited for gluelump Green's function in [21]

$$
G_{\mu \nu}(x, y)=\operatorname{tr}_{a} \int_{0}^{\infty} d s(D z)_{x y} e^{-K}\left\langle W_{\mu \nu}^{(F)}\left(C_{x y}\right)\right\rangle
$$

where $K=\frac{1}{4} \int_{0}^{s}\left(\frac{d z_{\mu}}{d \tau}\right)^{2} d \tau$ and

$$
W_{\mu \nu}^{F}\left(C_{x y}\right)=P P_{F}\left\{\exp \left(i g \int A_{\lambda} d z_{\lambda}\right) \exp \left(2 i g \int_{0}^{s} d \tau \hat{F}_{\sigma \rho}(z(\tau))\right)\right\}_{\mu \nu}
$$

and the closed contour $C_{x y}$ is formed by the straight line from $y$ to $x$ due to the heavy adjoint source Green's function and the path of the valence gluon $a_{\mu}$ from $x$ to $y$. Note that the nontrivial $\{\mu \nu\}$ dependence of the r.h.s. 
of (15) occurs only due to the $\hat{F}_{\nu \rho}$, expanding in powers of this term, one has $W_{\mu \nu}^{F}=W^{(0)} \delta_{\mu \nu}+W^{(1)} \delta_{\mu \nu}+W^{(1)} \hat{F}_{\mu \nu}+\ldots$ Neglecting in $G_{\mu \nu}$ gluon fields altogether we obtain the perturbative result, $G_{\mu \nu} \rightarrow G_{\mu \nu}^{(0)}$

$$
G_{\mu \nu}^{(0)}(x, y)=\frac{N_{c}\left(N_{c}^{2}-1\right) \delta_{\mu \nu}}{4 \pi^{2}(x-y)^{2}}, \quad f^{(0)}=\frac{N_{c}\left(N_{c}^{2}-1\right)}{4 \pi^{2}(x-y)^{2}} .
$$

This is the leading term in the expansion of $G_{\mu \nu}$ at small $|x-y|$, while the higher order terms are given by the OPE formalism [25]

$$
G_{\mu \nu}(x, y)=G_{\mu \nu}^{(0)}(x, y)\left(1+C_{1} \alpha_{s} \ln |x-y|+\ldots\right)+\delta_{\mu \nu}\left(C_{0}(x-y)^{2}+C_{4}(x-y)^{4}+\ldots\right)
$$

where it is always assumed that $C_{0} \sim D_{1}(0) \sim\left\langle\operatorname{tr} F_{\mu \nu}^{2}(0)\right\rangle$ is finite. The analysis in 26] using also nonperturbative nonlocal operators is supporting the expansion (17). To test the behaviour of $G_{i k}(x, y)$ at small $(x-y)^{2}$, one should take into account that small Wilson loops have a typical limiting form [1, ${ }^{1}$

$$
\langle W\rangle=\exp \left(-\frac{g^{2}\left\langle t r F^{2}(0)\right\rangle}{24 N_{c}} S^{2}\right)
$$

where $S$ is the minimal area of the small loop,

$$
S^{2}=\int_{C} z_{\mu}(x) d x_{\nu} \int_{C} z_{\mu}\left(x^{\prime}\right) d x_{\nu}^{\prime} \approx \int z_{\mu}^{2}(t) d t T
$$

where $T \equiv|x-y|$. Introducing (18) into (14) one obtains the path integral representation

$$
G_{i k}(x, y)=\delta_{i k} \int_{0}^{\infty} d s(D z)_{x y} \exp \left\{-\frac{1}{4} \int_{0}^{s} \dot{z}_{\mu}^{2}(\tau) d \tau-\omega^{2} \int_{0}^{s} z_{\mu}^{2} d \tau\right\}
$$

which can be estimated at small $|x-y|$, as it is explained in Appendix 1 . The result is

$$
\left.G_{i k}(x, y)\right|_{|x-y| \rightarrow 0} \approx \frac{N_{c}\left(N_{c}^{2}-1\right)}{4 \pi^{2} T^{2}}\left(1-\frac{\omega^{2} T^{2}}{4}+\ldots\right)
$$

with $\omega^{2}=\frac{g^{2}}{12 N_{c}}\left\langle\operatorname{tr} F^{2}\right\rangle T^{2}$, which yields for $D_{1}$ according to (13),

$$
D_{1}(x)=\frac{4 C_{2} \alpha_{s}}{\pi}\left\{\frac{1}{x^{4}}+\frac{\pi^{2} G_{2}}{24 N_{c}}+\ldots\right\},
$$

\footnotetext{
${ }^{1}$ We do not discuss here the renormalization properties of the r.h.s. of (18), since as it is seen in (22), those reduce to the renormalization of gluonic condensate, widely discussed in the framework of the OPE and ITEP sum rules [25].
} 
where $G_{2}$ is the standard gluonic condensate [25]

$$
D(0)+D_{1}(0)=\frac{g^{2}}{12 N_{c}} \operatorname{tr} F^{2}(0)=\frac{\pi^{2}}{18} G_{2}
$$

One can check consistency of the resulting $D_{1}(x)$. First one considers the singular term, $D_{1}^{s i n g}(x)=\frac{4 C_{2} \alpha_{s}}{\pi x^{4}}$ and inserts it in the static $Q \bar{Q}$ potential. The static $Q \bar{Q}$ potential can be expressed through $D$ and $D_{1}$, as was done in [27].

$$
V(r)=2 r \int_{0}^{r} d \lambda \int_{0}^{\infty} d \nu D(\lambda, \nu)+\int_{0}^{r} \lambda d \lambda \int_{0}^{\infty} d \nu\left[-2 D(\lambda, \nu)+D_{1}(\lambda, \nu)\right]
$$

Inserting in (24) the perturbative part of $D_{1}$ from (22) one obtains the standard color Coulomb potential $V(r)=-\frac{4 \alpha_{s}}{3 r}$, thus checking the correct normalization of $D_{1}(x)$.

Coming now to the constant term in (22) one can compare $D_{1}(0)$ on the l.h.s. of (22) with the r.h.s., $D_{1}(0)=\frac{\alpha_{s} C_{2}}{\pi} \cdot \frac{\pi^{2}}{18} G_{2}=\frac{\alpha_{s} C_{2}}{\pi}\left(D(0)+D_{1}(0)\right)$, where $D(0)+D_{1}(0)$ on the r.h.s. of (22) are defined by the gluon condensate, Eq. (23). Since $\frac{\alpha_{s} C_{2}}{\pi} \lesssim 1$, this estimate of $D_{1}(0)$ is reasonable and suggests that for $\alpha_{s}=0.4$ the magnitude of $D_{1}(0)$ is $0.2 D(0)$.

This ratio is in agreement with the lattice calculations in [6].

Another form of $G_{i k}(x, y)$ is available at all distances and practically important at large $|x-y|$, namely

$$
G_{i k}(x, y)=N_{c}\left(N_{c}^{2}-1\right) \sum_{n=0}^{\infty} \Psi_{n}^{(i)}(0) \Psi_{n}^{(k)^{+}}(0) e^{-M_{n}|x-y|}
$$

where $\Psi_{n}^{(i)}(x), M_{n}$ are eigenfunction and eigenvalue of the gluelump Hamiltonian, which is derived using the FFS path integral [21], details are given in Appendices 1,2,3,4 for the convenience of the reader.

$$
H^{(\text {glump })}=H_{0}^{(\text {glump })}+H_{\text {spin }}+H_{C} .
$$

Omitting the spin-splitting term $H_{\text {spin }}$ and the pertubative gluon exchange term $H_{C}$, which provide small corrections to the main term, one has for $H_{0}^{(\text {glump })}$ [21]

$$
H_{0}^{(\text {glump })}=\frac{\mu}{2}+\frac{p_{r}^{2}}{2 \mu}+\frac{L(L+1) / r^{2}}{2\left(\mu+\int_{0}^{1} d \beta \beta^{2} \nu(\beta)\right)}+\frac{\sigma_{a d j}^{2} r^{2}}{2} \int_{0}^{1} \frac{d \beta}{\nu(\beta)}+\int_{0}^{1} \frac{\nu(\beta)}{2} d \beta .
$$


Here $\mu, \nu$ are the so-called einbein functions to be found exactly from the stationary point of the Hamiltonian, $\frac{\delta H}{\delta \mu}=\frac{\delta H}{\delta \nu}=0$, or approximately [3, 27] from the stationary point of eigenvalues, e.g. $\frac{\delta M_{0}}{\delta \mu}=\frac{\delta M_{0}}{\delta \nu}=0$. In what follows we shall be interested in the case $L=0$, when $H_{0}$ reduces to

$$
H_{0}^{(\text {glump })}=\frac{\mu}{2}+\frac{p_{r}^{2}}{2 \mu}+\sigma_{a d j} r \rightarrow \sqrt{\mathbf{p}^{2}}+\sigma_{a d j} r .
$$

For $\Psi_{n}^{(\mu)}(0)$ one can use the known equation [28], which is obtained from the eigenfunctions $\Psi_{n}$ of $H_{0}$ through the connection [29]

$$
\Psi_{n}^{(\mu)}=\frac{e_{\mu}}{\sqrt{2 \mu}} \psi_{n}, \quad\left(\Psi_{n}^{(\mu)}(0)\right)^{2}=\frac{\sigma_{a d j}}{4 \pi} .
$$

Inserting (29) into (25) one obtains

$$
G_{\mu \nu}(x, y) \approx N_{c}\left(N_{c}^{2}-1\right) \sum_{n=0}^{\infty} \delta_{\mu \nu} \frac{\left(\sigma_{a d j}\right)}{4 \pi} e^{-M_{n}|x-y|} .
$$

It is clear that for $x \rightarrow y$ the sum in (30) diverges and one should use instead of (30) the perturbative answer (16). For large $|x-y|$ one can keep in (30) only the terms with the lowest mass, i.e. for the color electric gluelump state $1^{--}$, which obtains for spacial $\mu, \nu=i, k$

Thus one gets

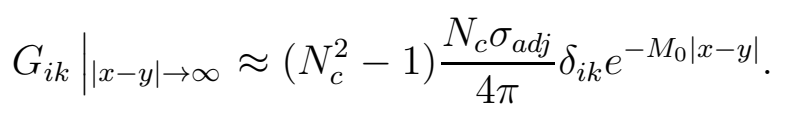

The eigenvalue $M_{0}$ was found in [21] to be $M_{0} \cong 1.5 \mathrm{GeV}$ for $\sigma_{f}=0.18$ $\mathrm{GeV}^{2}$.

Using (13) one can define from (31) the nonperturbative part of $D_{1}$, which is valid at large $|x|$,

$$
D_{1}^{(n o n p)}(x)=\frac{C_{2}(f) \alpha_{s} 2 M_{0} \sigma_{a d j}}{\sqrt{x^{2}}} e^{-M_{0}|x|}, \quad C_{2}(f)=\frac{N_{c}^{2}-1}{2 N_{c}}
$$

and the total $D_{1}$ due to (13) and (16) can be represented as

$$
D_{1}(x)=\frac{4 C_{2}(f) \alpha_{s}}{\pi x^{4}}+O\left(\alpha_{s}^{2}\right)+D_{1}^{(n o n p)}(x)
$$




\section{The correlator $D(x)$}

We now turn to the function $D(x)$ and to this end we specify the indices fo $D_{\mu \nu, \lambda \sigma}$ in (6) as $D_{i k, l m}(x, y)$ with $i, k, l, m=1,2,3$ and take the interval $h_{\mu}=x_{\mu}-y_{\mu}$ to lie on the temporal axis, $h_{4} \neq 0, h_{i} \equiv 0, i=1,2,3$. One can again represent $D_{i k, l m}$ as in (6),

$$
D_{i k l m}(x, y)=D_{i k, l m}^{(0)}+D_{i k, l m}^{(1)}+D_{i k, l m}^{(2)}
$$

As in the previous section, $D^{(0)}$ contributes to the function $D_{1}$, while the

contour differention operation $\overleftarrow{\delta}_{\mu}$ introduces the new field $F_{\mu 4}$, so that one has to do with the triple correlator $\operatorname{tr}\langle F \Phi F \Phi F\rangle$. In this paper we are considering the Gausian approximation for simplicity and neglect all correlators except for the quadratic ones, Eq. (1). Therefore the term $D^{(0)}$ and $D^{(1)}$ in (34) do not contribute to $D(x)$, and we concentrate on the last term in (34), $D^{(2)}$, which can be written as

$$
D_{i k, l m}^{(2)}(x, y)=-\frac{g^{4}}{2 N_{c}^{2}}\left\langle\operatorname{tr}_{a}\left(\left[a_{i}, a_{k}\right] \hat{\Phi}(x, y)\left[a_{l}, a_{m}\right]\right)\right\rangle .
$$

This function can be connected to the two-gluon gluelump Green's function. The two gluon gluelumps considered in [21] belong to the symmetric in color and spin components, while here one can rewrite (35) as

$$
\left[a_{i}, a_{k}\right]=i a_{i}^{a} a_{k}^{b} f^{a b c} T^{c}
$$

and the resulting gluelump function is

$$
G_{i k, l m}=t r_{a}\left\langle f^{a b c} f^{d e f} a_{i}^{a}(x) a_{k}^{b}(x) T^{c} \hat{\Phi}(x, y) T^{f} a_{l}^{d} a_{m}^{e}\right\rangle .
$$

One can immediately see that the gluelump in (37) is antisymmetric both in color and spin indices, but the total wave function is symmetric and the relative angular momentum $L$ of the lowest state can be taken as $L=0$. One can fix in (37) color indices $a, b ; d, e$ and average the Green's function over all fields $A_{\mu}^{h}$ with $h \neq a, b ; d, e$. Using the same argument as it was done for the one-gluon gluelump function, one can argue that this averaging will produce the white string (of triangle shape at any given moment), and hence it will ensure terms $\left(\delta_{a d} \delta_{b e}+\right.$ permutations $)$. As a result one can represent $G_{i k, l m}$ in the form

$$
G_{i k, l m}(x, y)=N_{c}^{2}\left(N_{c}^{2}-1\right)\left(\delta_{i l} \delta_{k m}-\delta_{i m} \delta_{k l}\right) G^{(2 g l)}(x, y)
$$


where $G^{(2 g l)}(x, y)$ is the Green's function of the two-gluon gluelump, which was studied in [21], and the Hamiltonian and lowest eigenvalue was found there explicitly (see also Appendix A of [21]).

Comparison of Eqs. (11), (35) and (38) immediately yields the following expression for $D(x)$.

$$
D(x-y)=\frac{g^{4}\left(N_{c}^{2}-1\right)}{2} G^{(2 g l)}(x, y)
$$

If one exploits for $G^{(2 g l)}$ the perturbative expression

$$
G^{(2 g l)(0)}(x, y)=\frac{1}{\left(4 \pi^{2}(x-y)^{2}\right)^{2}}+O \frac{\left(\alpha_{s} \ln (x-y)\right)}{(x-y)^{4}}
$$

then one recovers the corresponding perturbative expansion for $D(x)$, which was studied in [29]. However, as it was shown in [30, all perturbative terms of $D(x)$ are cancelled by those of higher correlators, so that they do not contribute the (divergent) terms in the expression for the string tension

$$
\sigma=\frac{1}{2} \int d^{2} x(D(x)+\text { higher correlators }) \text {. }
$$

Nonperturbative contribution to $D(x)$ can be written down using the spectral decomposition for $G^{(2 g l)}(T), T=x_{4}-y_{4}$,

$$
G^{(2 g l)}(T)=\sum\left|\Psi_{n}^{(2 g l)}(0)\right|^{2} e^{-M_{n}^{(2 g l)} T}
$$

Here $\Psi_{n}^{(2 g l)}(\boldsymbol{\xi}, \boldsymbol{\eta})$ is the two-gluon gluelump wave function calculated with the string Hamiltonian, neglecting spin-spin interaction in the first approximation, considered in 21], $\boldsymbol{\xi}, \boldsymbol{\eta}$ are Jacobi coordinates in the system of two gluons and the adjoint fixed center.

The calculation of $M_{n}^{(2 g l)}$ and $\Psi_{n}^{(2 g l)}(0)$ is discussed in the Appendix 2, and here we quote the final result for the lowest gluelump state:

$$
M_{0}^{(2 g l)}=2.56 \mathrm{GeV}, \quad\left|\Psi_{n}^{(2 g l)}(0)\right|^{2}=0.108 \sigma_{f}^{2} .
$$

Hence the leading at large $T$ asymptotics of $D(x)$ is

$$
D^{(2 g l)}(x) \cong \frac{g^{4}\left(N_{c}^{2}-1\right)}{2} 0.108 \sigma_{f}^{2} e^{-M_{0}^{(2 g l)}|x|}, \quad M_{0}^{(2 g l)}|x| \gg 1
$$


The corresponding value of the gluon correlation length is very small $T_{g}^{(2 g)}=\frac{1}{M_{0}^{(2 g l)}} \cong 0.08 \mathrm{fm}$, and different from the correlation length of $D_{1}(x)$, which is $T_{g}^{(1 g)} \equiv \frac{1}{M_{0}^{(19 l)}}=0.13 \mathrm{fm}$. This fact is in contradiction with the lattice calculations of the Pisa group in [6], where both correlation lengths coincide. To understand the reason for this discrepancy one must consider the higher in $\alpha_{s}$ terms contributing to $D(x)$. Indeed, using the term $L_{3}$ in the Lagrangian, which transforms two-gluon state into one-gluon and three-gluon states, one obtains in $D(x)$ the one-gluon state with the same asymptotics as in $D_{1}(x)$, namely

$$
D(x)=D^{(2 g l)}(x)+C_{1} \alpha_{s}^{3} D^{(1 g l)}(x)+C_{2} \alpha_{s}^{3} D^{(3 g l)}(x)+\ldots
$$

Here $D^{(1 g l)}(x) \sim \exp \left(-M_{0}^{(1 g l)}|x|\right), \quad D^{(3 g l)}(x) \sim \exp \left(-M_{0}^{(3 g l)}|x|\right)$. In a similar way $D_{1}(x)$ acquires terms with two-gluon and three-gluon gluelump asymptotics. Still effectively one expects that correlation lengths satisfy $T_{g}\left(D_{1}\right)>T_{g}(D)$, and this in agreement with lattice calculations of Bali et al (last ref. in [6]).

\section{Discussion of results}

In (32), (33) and (44) we have obtained the perturbative and nonperturbative parts of $D_{1}(x)$ and $D(x)$ and this is the main result of the paper.

Coming back to the three basic points outlined in the Introduction, the first point refers to the distinction between perturbative and nonperturbative and the essence of the nonperturbative mechanism of confinement. In this regard one should consider the mechanism which creates massive gluelump, namely via the path-integral representation (14) and using Wilson loop area law one obtains the Hamiltonian (27), where nonperturbative dynamics is connected to the string tension $\sigma$. The latter in its turn is expressed through $D(x)$ as in (41). Thus the signature for the nonperturbative is $\sigma \neq 0$, and one can separate from $D(x), D_{1}(x)$ perturbative parts as the limit of $D(x)$, $D_{1}(x)$ when $\sigma$ tends to zero.

One should stress here that it would be in general incorrect to represent $D(x), D_{1}(x)$ as a sum of perturbative and nonperturbative components. The expansion (45) gives instead another type of representation where the perturbative and nonperturbative are strongly mixed up. One can only state, that 
at small distances correlators behave perturbatively, while at large distances decay nonperturbatively.

At this point it is necessary to ask, what is the nonperturbative mechanism which creates $D(x)$ and according to (41) also creates confinement itself.

The answer lies in our separation of (3), where in contrast to the usual background formalism [22], both fields $a_{\mu}^{a}$ and $B_{\mu}^{b}$ are intrinsically the same, but the fields $B_{\mu}^{b}$ with color index $b \neq a$ are assumed to form the collective field which after averaging acts as a "white force" on the gluon $a_{\mu}^{a}$ with the correlators $D(x), D_{1}(x)$ as a measure of this force. As a result the gluon $a_{\mu}^{a}$ forms the bound state, which can be used to calculate $D(x), D_{1}(x)$. In this way the problem becomes self-consistent, when one can ensure that the same $D(x), D_{1}(x)$ act as input in the "white force" and result in the outcome of calculation of correlators. In the present paper this selfconsistency was checked only partially on the level of the string tension $\sigma$.

At the same time the selfconsistency allows one to connect $\Lambda_{Q C D}$ and $\sigma$, thus reducing the number of parameters in theory to one, as it should be in QCD.

Indeed, calculating $\sigma_{f}$ via (41) and using (44) for $D(x)$ one obtains

$$
\sigma_{f}=\left(\frac{\alpha_{s}}{0.3}\right)^{2} 0.53 \sigma_{f}
$$

where $\sigma_{f}$ on the r.h.s. is coming from the input $D(x)$, while that on the l.h.s. is from the resulting $D(x)$. For reasonable values of $\alpha_{s} \sim 0.41$ one obtains that $D^{(2 g l)}(x)$ satisfies the selfconsistency criterium (46). Neglecting all other contributions to $D(x)$ in (45), one can consider (46) as an equation defining the connection between $\sigma_{f}$ and $\Lambda_{Q C D}$. Namely writing $\alpha_{s}=\alpha_{s}\left(M_{0}^{(2 g l)}\right)=$ $0.41=\frac{4 \pi}{\beta_{0} \ln \left(\frac{M_{0}^{(2 g l)}}{\Lambda_{Q C D}}\right)^{2}}$, one finds

$$
\Lambda_{Q C D}\left(n_{f}=0\right)=0.25 M_{0}^{(2 g l)}=0.88 \sqrt{\sigma_{f}},
$$

and for $\sigma_{f}=0.18 \mathrm{GeV}^{2}$ one has $\Lambda_{Q C D}=0.375 \mathrm{GeV}$. One should compare this result with the value of $\Lambda_{Q C D}^{(\overline{M S})}$, obtained on the lattice (see e.g. [32] and refs. there), $\Lambda_{Q C D}^{(\overline{M S})}\left(n_{f}=0\right)=0.242 \mathrm{GeV}$, which is easily recalculated for $\Lambda_{Q C D}^{(V)}=(385 \pm 30) \mathrm{MeV}$, in good agreement with our theoretical value. 
Keeping in $D(x)$ also higher order terms, as in (45) and inserting in (41) one obtains instead of (46) an expansion

$$
\sigma_{f}=\left(\frac{\alpha_{s}}{0.41}\right)^{2} \sigma_{f}+C_{1} \alpha_{s}^{3} \sigma_{f}+. .
$$

In this way one finds a general type of connection between $\sigma_{f}$ and $\Lambda_{Q C D}$, as was stated in the point 3 of Introduction. Coming now to the small $x$ behaviour in (44) it should be replaced by an analytic one, $D(x) \sim c_{0}+$ $c_{1} x^{2}+\ldots$, as it follows from [31, and the gluonic condensate calculated from $D^{(2 g l}(0)$ in (44)), which is much larger than the standard value [25], will be

replaced by a smaller value, and in addition also $D^{(1 g l)}(x)$ in (45) contributes to $\sigma_{f}$. Therefore a more detailed analysis, including the behaviour of $D(x)$ at small $x$ is required, which is relegated to future publications.

Summarizing the results, we have computed the first terms of perturbative and nonperturbative field correlators $D(x)$ and $D_{1}(x)$ in the expansion in powers of $\alpha_{s}$ with coefficient functions proportional to the gluelump Green's function, and have made the first check of selfconsistency of the resulting string tension. In this way the preliminary analysis in the paper supports the confinement mechanism as the formation of the selfconsistent background field acting as a white string on propagating gluons.

The author is grateful to A.M.Badalian and Yu.S.Kalashnikova for fruitful discussions, and V.I.Shevchenko for collaboration at the first stages of the work.

The work is supported by the Federal Program of the Russian Ministry of industry, Science and Technology No.40.052.1.1.1112, and by the grant for scientific schools NS-1774. 2003. 2.

\section{Appendix 1}

Derivation of Eq. (7)

Consider the general bilocal correlator,

$$
\Psi(x, y) \equiv\left\langle\operatorname{tr} K_{1}(x) \hat{\Phi}(x, y) K_{2}(y)\right\rangle
$$


where $\hat{\Phi}(x, y)$ is the adjoint parallel transporter,

$$
\hat{\Phi}(x, y)=P \exp i g \int_{y}^{x} \hat{A}_{\mu} d z_{\mu}
$$

Taking the difference

$$
\Delta_{\mu} \Psi(x, y) \equiv \Psi\left(x+\delta x_{\mu}, y\right)=\left\langle\operatorname{tr} K_{1}\left(x+\delta x_{\mu}\right) \hat{\phi}\left(x+\delta x_{\mu}, y\right) K_{2}(y)\right\rangle-\Psi(x, y)
$$

one can deform identically the shifted contour by insertion of double lines of the $L$ form $N$ times at distance $a$ from each other, $N a=|x-y|$. The explicit form of deformation is shown in Fig.2 of the last ref. [23]. In the limit when $\delta x_{\mu} \rightarrow 0$, and $a \rightarrow 0, N \rightarrow \infty$, any open $(a \times a)$ plaquette from two neighboring $L$ insertions tends to $\left(F_{\mu \lambda} a^{2}\right)$ and one obtains

$\frac{\partial_{\mu} \Psi(x, y)}{\partial x_{\mu}}=\left\langle\operatorname{tr}\left(\frac{\partial K_{1}(x)}{\partial x_{\mu}}-i g K_{1}(x) \hat{A}_{\mu}(x)\right) \hat{\Phi}(x, y) K_{2}(y)\right\rangle+\left\langle\operatorname{tr} K_{1}(x) \delta_{\mu}(x) \hat{\Phi}(x, y) K_{2}(y)\right\rangle$

where $\delta_{\mu}(x)$ is defined in Eq.(8).

\section{Appendix 2}

\section{Calculation of $D_{1}(x)$ at small $x$}

One must calculate the path integral for the gluon Green's function

$$
G_{i k}(x, y)=\delta_{i k} \int d s(D z)_{x y} e^{-K}\langle W\rangle
$$

where $\langle W\rangle$ is given in (18) and $K=\frac{1}{4} \int_{0}^{s} \dot{z}_{\mu}^{2}(\tau) d \tau$.

Making an approximation (19) and introducing the dynamical mass variable $\mu(t)=\frac{d t}{2 d \tau}$, where $\tau \leq s$ in the proper time [2, 3], one can replace $d s\left(D z_{4}\right)_{x_{4}, y_{4}}$ by the path integral $D \mu$ see 35] for more details) as follows

$$
G_{i k}(0 T)=\delta_{i k} \int \frac{1}{2 \bar{\mu}}\left(D^{3} z\right)_{0,0} D \mu e^{-\int_{0}^{T} d t\left(\frac{\mu}{2}+\frac{\mu \dot{\mathbf{Z}}^{2}}{2}+\frac{\mu \omega^{2} \mathbf{Z}^{2}}{2}\right)}
$$


where we have defined

$$
\frac{\mu \omega^{2}}{2}=\frac{g^{2}}{24 N_{c}}\left\langle\operatorname{tr} F_{\mu \nu}^{2}\right\rangle T,\left(D^{3} z\right) D \mu=\prod_{n=1}^{N} \frac{d^{3} \Delta z(n)}{l^{3}(n)} \frac{d \mu(n)}{l_{\mu}(n)},
$$

and

$$
\begin{gathered}
l_{\mu}(n)=\left(\frac{2 \pi \mu(n)}{\Delta t}\right)^{1 / 2}, l(n)=\left(\frac{2 \pi \Delta t}{\mu(n)}\right)^{1 / 2}, \Delta t N=T, \quad N \rightarrow \infty . \\
\bar{\mu}=\frac{1}{s} \int_{0}^{s} \mu(\tau) d \tau \approx \frac{1}{T} \int_{0}^{T} \mu(t) d t .
\end{gathered}
$$

The integration over $\left(D^{3} z\right)_{00}$ in (A2.2) is known from the textbook solution for the Green's function of oscillator, hence one has

$$
G_{i k}(0 T)=\delta_{i k} \int \frac{D \mu}{2 \bar{\mu}} e^{-\int_{0}^{T} d t \frac{\mu}{2}}\left(\frac{\bar{\mu} \omega}{2 \pi \operatorname{sh} \omega T}\right)^{3 / 2}=\frac{1}{2 \bar{\mu}}\left(\frac{\bar{\mu} \omega}{2 \pi s h \omega T}\right)^{3 / 2}
$$

Now one can obtain $\bar{\mu}$ from the solution of the free Green's function at small $T$, which is realized when $\omega \rightarrow 0$,

$$
\frac{1}{2 \bar{\mu}}\left(\frac{\bar{\mu}}{2 \pi t}\right)^{3 / 2}=\frac{1}{4 \pi^{2} T^{2}}, \quad \bar{\mu}=\frac{2}{\pi T}
$$

and expanding further (A2.3), one finally obtains

$$
G_{i k}=\frac{1}{4 \pi^{2} T^{2}}\left(1-\frac{\omega^{2}}{4} T^{2}\right)
$$

and using relation (13) one finds resulting equation (22) for $D_{1}(x)$, where

$$
G_{2} \equiv \frac{2 \alpha_{s}}{\pi}\left\langle t r F_{\mu \nu}^{2}\right\rangle
$$

\section{Appendix 3}

\section{Derivation of the gluelump Hamiltonian}


One starts with the expressions (14), (15) for the gluelump Green's function, obtained in the framework of the FFS formalism [24]. The first step is the vacuum averaging of $\left\langle W_{\mu \nu}^{F}\right\rangle$, which is done using the nonabelian Stokes theorem and the cluster expansion where we systematically keep the lowest order (quadratic) terms in powers of field correlators, as is explained in the Introduction. The result is

$$
\left\langle W_{\mu \nu}^{F}\right\rangle=\exp \left\{-\frac{\xi}{2} \int d \rho_{\rho \lambda}(u, \tau) \int d \rho_{\rho^{\prime} \lambda^{\prime}}\left(u^{\prime}, \tau^{\prime}\right) D_{\rho \lambda, \rho^{\prime} \lambda^{\prime}}\left(u, u^{\prime}\right)\right\}
$$

where we have defined

$$
\xi \equiv \frac{C_{2}(a d j)}{C_{2}(f)}, \quad d \rho_{\rho \lambda}(u, \tau)=d \sigma_{\rho \lambda}(u)-2 i \hat{S}_{\rho \lambda} d \tau
$$

and $d \sigma_{\rho \lambda}(u)$ is the surface element, while $\hat{S}_{\rho \lambda}$ contains gluon spin operators and prescription of replacing the coordinate $u$ of the surface element by the coordinate of the boundary - gluon trajectory $z_{\mu}(\tau)$,

$$
\hat{S}_{\rho \lambda} F_{\rho \lambda}(u)=\left(S_{i} H_{i}+\tilde{S}_{i} E_{i}\right) \delta_{u, z(\tau)}
$$

In what follows we shall not use the explicit form of $S_{i}, \tilde{S}_{i}$.

In (A3.1) three types of terms are present:

a terms quadratic in $d \sigma$, which yield the area law for large area, much larger than $T_{g}^{2}$;

b mixed term, proportional to $d \sigma_{\rho \lambda} \tilde{S}_{\rho \lambda}$. These produce spin-dependent terms in the Hamiltonian, as it is explained in [21, [27].

c terms quadratic in $\hat{S}$; they give rise to the self-energy terms of the propagating gluon and are treated in Appendix 3. It is shown there, that the self-energy terms occurring due to both quartic terms $a^{4}$ in the QCD Lagrangian and terms quadratic in $\hat{S}$ should cancel in the framework of BFF.

As a result, if one neglects spin-dependent terms in the first approximation, one can keep in (A3.1), (A3.2) only the surface element, $d \rho \rightarrow d \sigma$, and the r.h..s. of (A3.1) simplifies, namely

$$
\left\langle W_{\mu \nu}^{F}\right\rangle=\delta_{\mu \nu} \exp \left(-\sigma_{a d j} A\right)
$$


where $A$ is the area between the gluon path $z(\tau), \tau \epsilon[0, s]$, and the piece of the straight line between $x$ and $y$. It is usually assumed on physical grounds (and supported by lattice data) that $A$ is the minimal area for the given boundary.

As a next step one should write the resulting expression for the gluelump Green's function, with gluon spin effects neglected,

$$
G_{\mu \nu}^{(0)}(x, y)=\delta_{\mu \nu} \int_{0}^{\infty} d s(D z)_{x y} e^{-K-\sigma_{a d j} A}, \quad K=\frac{1}{4} \int_{0}^{s} d \tau\left(\dot{z}_{\mu}\right)^{2}
$$

in the form, where real time $t$ instead of the proper time $\tau, s$ enters.

This is done in Appendix2, Eq. (A2.2) where the relation is used $d s\left(D^{4} z\right)_{x y}=$ $\left(D^{3} z\right) \frac{D \mu}{2 \bar{\mu}}$, and explicit expressions for $\bar{\mu}, D \mu$ are given. Here $\mu(t)$ connects real (Euclidean) time $t$ and proper time $\tau, 2 \mu(t)=\frac{d t}{d \tau}$. The transformations discussed below have been introduced in 36 .

One can write the area $A$ in the Nambu-Goto form,

$$
A=\int_{0}^{1} d \beta \int_{0}^{T} d t \sqrt{\dot{w}_{i}^{2} w_{k}^{\prime 2}-\left(\dot{w}_{i} w_{i}^{\prime}\right)^{2}}, \quad T=\left|x_{4}-y_{4}\right|,
$$

with $w(\beta, t)$ being the coordinate on the string world sheet, $\dot{w}_{i} \equiv \frac{\partial w_{i}}{\partial t}, \quad w_{i}^{\prime} \equiv$ $\frac{\partial w_{i}}{\partial \beta}$.

One can approximate the minimal area choosing $w_{i}(\beta)$ in the form of the piece of the straight line, $w_{i}(\beta, t)=r_{i}(t) \beta$.

One may wonder what is the accuracy of the straight-line approximation, i.e. of the neglecting the string excitation, which in the background field formalism amounts to the hybrid excitations, with the energy gap of around $1 \mathrm{GeV}$. Therefore all nonadiabatic effects of this type are typically of the order of the hybrid admixture to the given gluelump state. For the ground state this admixture was calculated and discussed in [37, and appeared to be of the order of few percent.

As a next step one introduces einbein variables to get rid of the square root in (A2.6), and as a result the total Euclidean action $\mathcal{E}$ as a function of einbein parameters $\mu(t), \eta(t), \nu(t)$ has the form

$$
\mathcal{E}(\mu, \eta, \nu)=\int_{0}^{T} d t \int_{0}^{1} d \beta\left[\frac{\mu \dot{r}_{i}^{2}}{2}+\frac{1}{2 \nu}\left(\dot{w}_{i}^{2}+\left(\sigma_{a d j} \nu\right)^{2} r^{2}-2 \eta\left(\dot{w}_{i} r_{i}\right)+\eta^{2} r^{2}\right)\right]
$$

Finally the gluelump Green's function can be written as 


$$
G_{\mu \nu}^{(0)}(x, y)=\delta_{\mu \nu} \int D^{3} r_{i} D \nu D \eta \frac{D \mu}{2 \bar{\mu}} e^{-\mathcal{E}}
$$

From (A2.8) integrating over $D \eta$ one can go over to the Minkowskian Hamiltonian in the standard way using the Feynman prescription:

$$
\int(D x)_{i f} e^{i a c t i o n}=\left\langle i\left|e^{-i H T}\right| f\right\rangle
$$

The Hamiltonian $H=H(\nu, \mu)$ depends on the auxiliary einbein functions $\nu, \mu$ and the remaining integral over $D \nu D \mu$ can be taken using the stationary point method. The gluelump Hamiltonian $H(\nu, \mu)$ has the form [21], given in Eq. (27) of the main text

$$
H^{(\text {glump })}=\frac{\mu}{2}+\frac{p_{r}^{2}}{2 \mu}+\frac{L(L+1)}{2 I r^{2}}+\frac{\left(\sigma_{a d j} r\right)^{2}}{2} \int_{0}^{1} \frac{d \beta}{\nu(\beta)}+\int_{0}^{1} \frac{\nu(\beta)}{2} d \beta
$$

where the factor of inertia moment $I$ is

$$
I=\mu+\int_{0}^{1} d \beta \beta^{2} \nu(\beta)
$$

For $L=0$ the Hamiltonian is easily computed taking stationary point values of $\nu=\nu_{0}=\sigma_{a d j} r$ and $\mu=\mu_{0}=\sqrt{p_{r}^{2}}$,

$$
H(L=0)=\sqrt{p_{r}^{2}}+\sigma_{a d j} r
$$

The lowest eigenvalue of (A3.12) is easily calculated to be $M_{0} \approx 1.5 \mathrm{GeV}$. Note that perturbative interaction is not included in (A3.12), taking that into account the eigenvalue drops to $M_{0} \approx 1 \mathrm{GeV}$ for $\alpha_{s}(r) \approx \bar{\alpha}_{s} \approx 0.2$. Since this interaction is subject to strong radiative corrections of destructive character, we do not take it into account, see [21] for more discussion.

\section{Appendix 4}

The gluon self-energy and auxiliary function method for the effective Lagrangian of QCD 
One starts with the decomposition of the gauge field $A_{\mu}$ into background component $B_{\mu}$ and valence gluon component $a_{\mu}$,

$$
A_{\mu}=B_{\mu}+a_{\mu}
$$

We shall not specify below the principle of decomposition, one can use e.g. the idea of separating one or a group of few colors for $a_{\mu}$, while the rest of colors is in $B_{\mu}$, as it was done in [17, 38, which assumes a fixed gauge for $a_{\mu}$. The following derivations are of general character and do not depend on the assumed separation. Writing the QCD Lagrangian as

$$
L(A)=L(B+a)=\frac{1}{2} \operatorname{tr} F_{\mu \nu}^{2}(B+a),
$$

where $F_{\mu \nu}$ is

$$
\begin{gathered}
F_{\mu \nu}(x)=\partial_{\mu} A_{\nu}-\partial_{\nu} A_{\mu}-i g\left[A_{\mu}, A_{\nu}\right]= \\
=\partial_{\mu}\left(a_{\nu}+B_{\nu}\right)-\partial_{\nu}\left(a_{\mu}+B_{\mu}\right)-i g\left[a_{\mu}+B_{\mu}, a_{\nu}+B_{\nu}\right]= \\
=\hat{D}_{\mu} a_{\nu}-\hat{D}_{\nu} a_{\mu}-i g\left[a_{\mu}, a_{\nu}\right]+F_{\mu \nu}^{(B)}
\end{gathered}
$$

one obtains the effective action for the field $a_{\mu}, S_{\text {eff }}(a)$, after integrating out the fields $B_{\mu}$, namely

$$
\left\langle e^{-\int L(B+a) d^{4} x}\right\rangle_{B} \equiv e^{-S_{e f f}(a)}
$$

where

$$
S_{e f f}(a)=\int\langle L(B+a)\rangle_{B} d^{4} x-\frac{1}{2} \int d^{4} x \int d^{4} y\langle\langle L(x) L(y)\rangle\rangle+\ldots
$$

In what follows we shall be interested in the terms of the fourth order in $a_{\mu}$, which appear in the path-integral representation of the valence gluon Green's function, namely [24]

$$
G_{\mu \nu} \equiv\left\langle a_{\mu}(x) a_{\nu}(y)\right\rangle_{a, B}=\left\langle\left(\hat{D}_{\lambda}^{2} \delta_{\mu \nu}-2 i g \hat{F}_{\mu \nu}\right)_{x, y}^{-1}\right\rangle_{B}
$$

where the background Feynman gauge is assumed for $a_{\mu}, D_{\mu}(B) a_{\mu}=0$.

Writing (A4.6) as a path integral, one has

$$
G_{\mu \nu}(x, y)=\int_{0}^{\infty} d s(D z)_{x y} e^{-K_{0}} \Phi_{\mu \nu}(x, y)
$$


where we have defined

$$
\begin{gathered}
K_{0}=\frac{1}{4} \int_{0}^{\infty}\left(\frac{d z_{\mu}}{d \tau}\right)^{2} d \tau ; \quad \Phi_{\mu \nu}(x, y)=\left[P_{F} P_{A} \exp \left(i g \int_{y}^{x} A_{\lambda} d z_{\lambda}\right) \times\right. \\
\left.\times \exp \left(2 g \int_{0}^{s} d \tau F_{\sigma \rho}(z(\tau))\right)\right]_{\mu \nu}
\end{gathered}
$$

The last exponential factor in (A4.9) represents the interaction of the valence gluon color magnetic moment with the background field, and the averaging of this factor over $B_{\mu}$ produces the effective self-energy term which is negative and which makes the gluon unstable (mass eigenvalues are not bounded from below). This is the paramagnetic instability of the relativistic charge moving in the Gaussian stochastic environment. A similar situation occurs for the quarks where the corresponding term has the form $\exp \left(g \sigma_{\mu \nu} \int_{0}^{s} d \tau F_{\mu \nu}(z(\tau))\right)$ and produces also the negative self-energy contribution, not bounded from below when considered outside of perturbation theory. In the quark case however, the term is a $4 \times 4$ Dirac matrix and one recovers the stability when considering also negative energy states (lower Dirac components) - see 39] for a discussion.

In the gluon case this mechanism is absent, and we now show that there is another stabilyzing phenomenon, associated with the gluon term $\sim a_{\mu}^{4}$ in the QCD Lagrangian.

To this end we consider two characteristic terms in the Lagrangian: the original quartic and another effective quartic generated by the paramagnetic term in $L(B+a)$ :

$$
L^{(F)}=g f^{i k a} a_{\mu}^{i} a_{\nu}^{k} F_{\mu \nu}^{a}(B)
$$

After averaging the square of $L^{(F)}$ as in the second term in (A4.5), one obtains the effective quartic term in the Lagrangian

$$
\left\langle e^{-L}\right\rangle_{\text {quartic }}=e^{-S_{\text {eff }}^{(4)}} ; S_{\text {eff }}^{(4)}=\int d^{4} x d^{4} y a_{\mu}^{a}(x) a_{\nu}^{b}(x) a_{\mu}^{a^{\prime}}(y) a_{\nu}^{b^{\prime}}(y) f^{a b c} f^{a^{\prime} b^{\prime} c} K(x, y)
$$

where we have defined

$$
K(x, y)=\frac{g^{2}}{4} \delta^{(4)}(x-y)-\frac{2 N_{c}}{N_{c}^{2}-1} D(x-y)
$$

and $D(x)$ is the standard field correlator [1, 2]

$$
g^{2}\left\langle\left\langle F_{\lambda \beta}(x) F_{\gamma \delta}(y)\right\rangle\right\rangle=\left(\delta_{\lambda \gamma} \delta_{\beta \delta}-\delta_{\lambda \delta} \delta_{\beta \gamma}\right) D(x-y)+O\left(D_{1}\right) .
$$


One can rewrite $S_{e f f}^{(4)}$ more conveniently as

$$
S_{e f f}^{(4)}=\int \Psi_{a a^{\prime}}(x, y) \tilde{K}_{a a^{\prime}, b b^{\prime}}(x, y) \Psi_{b b^{\prime}}(x, y) d^{4} x d^{4} y \equiv \Psi \tilde{K} \Psi
$$

and use the Hubbard-Stratonovich identity

$$
e^{-\Psi \tilde{K} \Psi}=\int \sqrt{\operatorname{det} \tilde{K}} D \chi e^{-\chi \tilde{K} \chi+i \Psi \tilde{K} \chi+i \chi \tilde{K} \Psi} .
$$

In (A4.14), (A4.15) we have used notations:

$$
\begin{gathered}
\tilde{K}_{a a^{\prime}, b b^{\prime}}(x, y)=f^{a b c} f^{a^{\prime} b^{\prime} c} K(x, y) \\
\Psi_{a a^{\prime}}(x, y)=a_{\mu}^{a}(x) a_{\mu}^{a^{\prime}}(y) \\
\chi \equiv \chi_{a a^{\prime}}(x, y)
\end{gathered}
$$

On the r.h.s. of (A4.15) the gluons $a_{\mu}$ enter only quadratically; combining quadratic terms from $L$ with the latter, one obtains the term in the partition function,

$$
Z=\int \exp \left(-S_{e f f}^{(2)}-\chi \tilde{K} \chi\right) D \chi
$$

with

$$
S_{\text {eff }}^{(2)}=\frac{1}{2} \int a_{\mu}^{a}(x)\left[-\hat{D}_{a b}^{2} \delta_{\mu \nu} \delta(x-y)-2 i I^{a b}(x, y)\right] a_{\nu}^{b}(y) d x d y
$$

where we have defined

$$
I^{a a^{\prime}}(x, y)=\tilde{K}_{a a^{\prime}, b b^{\prime}}(x, y) \chi_{b b^{\prime}}(x, y)
$$

After integrating out $D a_{\mu}$ one obtains an effective Lagrangian for the auxiliary fields $\chi$,

$$
Z \sim \int D \chi e^{-L_{e f f}(\chi)}, \quad L_{e f f}(\chi)=\frac{1}{2} \operatorname{tr} \ln \left(-D^{2}-2 i I\right)+\chi \tilde{K} \chi
$$

Using the stationary point method for the integral over $D \chi$, one has equations

$$
\left.\frac{\delta L_{e f f}(\chi)}{\delta \chi}\right|_{\chi=\chi^{(0)}}=0, \quad \chi^{(0)}=\frac{i}{2} \frac{1}{\left(-D^{2}-2 i I\right)}
$$


and defining the effective mass operator,

$$
\mathcal{M}_{0}^{2} \equiv-2 i I=-2 i \tilde{K} \chi^{(0)}
$$

one has equation for $\mathcal{M}_{0}^{2}$,

$$
\mathcal{M}_{0}^{2}=\tilde{K} \frac{1}{-D^{2}+\mathcal{M}_{0}^{2}}
$$

A nonzero solution for $M_{0}^{2}$ in (25) would imply the existence of the effective mass term of the valence gluon. At the same time the ghost Green's function does not have both quartic and magnetic moment contribution, so that for the ghost the effective mass is zero. To ensure the exact cancellation of the ghost and unphysical gluon degrees of freedom one must require that $\mathcal{M}_{0}^{2}$ vanish.

The r.h.s. of (A4.25) is ultraviolet divergent and needs renormalization, which can be accomplished to satisfy this requirement. Therefore we shall assume everywhere that the effective gluon mass is zero and the gluon magnetic moment term with $\hat{F}_{\sigma \rho}(z(\tau))$ in Eq. (15) is absent, as it was assumed in Appendix 3.

\section{Appendix 5}

\section{Spectral representation of the three-body Green's function}

In section 3 we have to evaluate the spectral representation of $G^{(2 g l)}(T)$ in (42) and the lowest eigenvalue $M_{0}^{(2 g l)}$. In this Appendix we give some details of derivation, using Appendix A of [21. The total eigenfunction of two-gluon gluelump $\Psi(\boldsymbol{\xi}, \boldsymbol{\eta})$ where $\boldsymbol{\xi}, \boldsymbol{\eta}$ are Jacobi coordinates,

$$
\boldsymbol{\eta}=\mathbf{r}_{12} / \sqrt{2}, \boldsymbol{\xi}=\left(\mathbf{r}_{1}+\mathbf{r}_{2}\right) / \sqrt{2}, \quad \rho^{2}=\boldsymbol{\xi}^{2}+\boldsymbol{\eta}^{2}
$$

can be expanded in the hyperspherical basis 33 .

$$
\Psi=\sum_{K, \nu} u_{K}^{\nu}(\Omega) \frac{y_{K}^{\nu}(\rho)}{\rho}
$$


where $y_{K}^{\nu}(\rho)$ is the hyperradial wave function and the series (A5.2) is fast converging for linear confinement (for a recent review and refs. see 34]), so that one can retain the lowest term in (A5.1) for the two-gluon gluelump corresponding to $K=0$. With the definition $u_{0}^{0}(\Omega)=\frac{1}{\sqrt{\pi^{3}}}, \quad y_{0}^{0}(\rho) \equiv y(\rho)$, the equation for $y(\rho)$ has the form

$$
y^{\prime \prime}+3 \frac{d}{d \rho}\left(\frac{y}{\rho}\right)+2 \mu(\varepsilon(\mu)-U(\rho)) y=0
$$

where $U(\rho)=C_{0} \sigma \rho, \quad C_{0}=\frac{32 \sqrt{2}(1+\sqrt{2})}{15 \pi}$, and the total mass eigenvalue is $M=\min (\mu+\varepsilon(\mu))$. Using the same approach as in [28], one can deduce the following relation

$$
|\psi(0)|^{2}=\frac{\mu}{8 \pi^{3}}\left\{\left\langle\frac{1}{\rho^{4}}\right\rangle 6(M-\mu)-5 \sigma C_{0}\left\langle\frac{1}{\rho^{3}}\right\rangle\right\}
$$

where $\left\langle\rho^{-n}\right\rangle=\int_{0}^{\infty} y^{2}(\rho) \rho^{5-n} d \rho$. From the minimization one can find the optimal $\mu=\mu_{0}=2.23 \sqrt{\sigma_{f}}$ and from the form of the effective potential, having minimum at $\rho=\rho_{0}=1.15 / \mu_{0}$, one can define with good accuracy that $\left\langle\rho^{-n}\right\rangle \approx \rho_{0}^{-n}$. Finally, taking into account the usual relativistic normalization of bosons, $\frac{1}{\sqrt{2 \mu}}$, the total normalization of the two-gluon wave-function appears as

$$
\left|\Psi_{n}(0)\right|^{2}=\frac{1}{4 \mu_{0}^{2}}|\psi(0)|^{2}=0.108 \sigma_{f}^{2}
$$

which was used in the main text.

The eigenvalue $M_{0}$ with the account of the hyperfine interaction reads (see 21] for details)

$$
M_{0}^{(2 g)}=6.15 \sqrt{\sigma_{f}}+\Delta M_{S S}
$$

with $\Delta M_{S S}=\mathbf{S}^{(1)} \mathbf{S}^{(2)} 0.49 \mu_{0} \frac{4}{3} \alpha_{s}$, and for $\mathbf{S}=\mathbf{S}^{(1)}+\mathbf{S}^{(2)}=\mathbf{1}$, and $\alpha_{s}=0.15$

one obtains the total eigenvalue, which is $M_{0}^{(2 g)}=6.03 \sqrt{\sigma_{f}}=2.56 \mathrm{GeV}$ for $\sigma_{f}=0.18 \mathrm{GeV}^{2}$.

\section{References}

[1] H.G.Dosch, Phys. Lett. B190 (1987) 177;

H.G.Dosch and Yu.A.Simonov, Phys. Lett. B205 (1988)339;

Yu.A.Simonov, Nucl. Phys. B307 (1988) 512 
[2] A.Di. Giacomo, H.G.Dosch, V.I.Shevchenko and Yu. A.Simonov, Phys.Rept., 372 (2002) 319, hep-ph/0007223

[3] Yu.A.Simonov, Yad. Fiz. 54 (1991) 192;

Yu.A.Simonov, in: "QCD: Perturbative or Nonperturbative?" eds. L.Ferreira., P.Nogueira, J.I.Silva-Marcos, World Scientific, 2001, hep-ph/9911237

[4] Yu.A.Simonov, Physics-Uspekhi, 39 (1996) 313

[5] D.S.Kuzmenko, V.I.Shevchenko, Yu.A.Simonov, Physics-Uspekhi, 174 (2003) 3

[6] A.Di Giacomo and H.Panagopoulos, Phys. Lett. B285, (1992) 133;

M.D'Elia, A.Di Giacomo, and E.Meggiolaro, Phys. Lett. B408, (1997) 315 ;

A.Di Giacomo, E.Meggiolaro and H.Panagopoulos, Nucl. Phys. B483, (1997) 371

M.D'Elia, A.Di Giacomo, and E.Meggiolaro, Phys. Rev. D67, (2003) 114504

G.S.Bali, N.Brambilla and A Vairo, Phys. Lett. B42 (1998) 265

[7] G.Bali, Phys. Rev. D62, 114503 (2000), hep-lat/0006022;

S.Deldar, Phys. Rev. D62, 034509 (2000), hep-lat/9911008

[8] Yu.A.Simonov, JETP Lett. 71, (2000) 127;

V.I.Shevchenko and Yu.A.Simonov, Phys. Rev. Lett. 85, (2000) 1811;

V.I.Shevchenko and Yu.A.Simonov, J. Mod. Phys. A; hep-ph/0104135

[9] A.M.Badalian and B.L.G.Bakker, Phys.Rev. D 66, (2002) 034025, hep-ph/0202246,

A.M.Badalian, B.L.G.Bakker and Yu.A.Simonov, Phys. Rev. D 66, (2002) 034026; hep-ph/0204088

[10] A.M.Badalian, V.P.Yurov,Yad. Fiz. 51, 1368 (1990); ibid. 56, 239 (1993);

A.M.Badalian, B.L.G.Bakker, Phys. Rev. D62, (2000) 094031;

A.M.Badalian, V.L.Morgunov, B.L.G.Bakker, Phys. Atom. Nucl. 63 (2000) 1635;

A.M.Badalian, A.I.Veselov, B.L.Bakker, Phys. Rev. D, (to appear); 
hep-ph/0411291

A.M.Badalian, B.L.G.Bakker, hep-ph/0412013]

[11] Yu.A.Simonov, in Proc. of the DAФNE Meeting, Frascati, 1991; Nucl. Phys. B (Proc. Suppl.) 23B, (1991) 283;

Yu.S.Kalashnikova and Yu.B.Yufryakov, Phys. Lett. B359, (1995) 175, Phys. Atom nucl. 60 (1997) 307

[12] Yu.S.Kalashnikova and D.S.Kuzmenko, Phys. Atom. Nucl. 64, (2001) 1716, ibid 67, (2004) 538

[13] Yu.A.Simonov, Z.Physik C53, (1992) 419;

Yu.S.Kalashnikova, A.V.Nefediev, Yu.A.Simonov Phys. Rev. D64, (2001) 014037; hep-ph/0103274;

Yu.A.Simonov, J.A.Tjon, Phys. Rev D (to appear); hep-ph/04099361

[14] A.B.Kaidalov, and Yu.A.Simonov, Phys. Lett. B477, (2000) 167;

hep-ph/9912434, Phys.Atom.Nucl. 63, (2000) 1428; hep-ph/991291

[15] M.Fabre de la Ripelle, Yu.A.Simonov, Ann. Phys. (NY) 212, (1991) 235;

B.O.Kerbikov and Yu.A.Simonov, Phys.Rev. 62, (2000) 093016;

Yu.A.Simonov, J.A.Tjon and J.Weda, Phys. Rev. D65 (2002) 0940013; hep-ph/0111344,

Yu.A.Simonov, Phys. Rev. D65, (2002) 116004; hep-ph/0203059;

Yu.A.Simonov, Phys. Atom. Nucl. 66 (2003) 338; hep-ph/0203059

[16] Yu.A.Simonov, Yad.Fiz. 58 (1995) 113;

Yu.A.Simonov, in: "Lecture Notes in Physics" H.Latal, W.Schweiger eds. Vol. 479, p. 138, Springer, 1996;

Yu.A.Simonov, Phys. Atom. Nucl. 65 (2002) 135, hep-ph/0109081;

Yu.A.Simonov, Phys. Atom. Nucl. 66 (2003) 764; hep-ph/0109159

[17] Yu.A.Simonov, Phys. At. Nucl. 61 (1998) 855, hep-ph/9712250

[18] Yu.A.Simonov, preprint IFUP-TH 52/92 (unpublished);

D.V.Antonov, Yu.A.Simonov, Int.J.Mod. Phys. A11 (1996) 4401

[19] I.Jorysz, C.Michael, Nucl. Phys. B302 (1988) 448;

N.Campbell, I.Jorysz, CMichael, Phys. Lett. B167 (1986) 91 
[20] M.Foster, C.Michael, Phys. Rev. D59 (1999) 094509, hep-lat/9811010

[21] Yu.A.Simonov, Nucl. Phys. B592 (2001) 350, hep-ph/0003114

[22] B.S.De Witt, Phys. Rev. 162, 1195, 1239 (1967)

J.Honerkamp, Nucl. Phys. B 48, 269 (1972);

G.'t Hooft Nucl. Phys. B 62, 444 (1973), Lectures at Karpacz, in : Acta Univ. Wratislaviensis 368, 345 (1976);

L.F.Abbot, Nucl. Phys. B 185, 189 (1981)

[23] L.Durand, E.Mendel, Phys. lett. B85, 241 (1979);

A.A.Migdal, Phys. Rep. 102, 200 (1983);

Yu.A.Simonov, Yad. Fiz. 50 (1989) 213

[24] Yu.A.Simonov and J.A.Tjon, Ann. Phys. (NY) 228,1 (1993);

Yu.A.Simonov and J.A.Tjon, in : M.S.Marinov Memorial Volume ed. by M.A.Olshanetsky and A.I.Vainshtein, World Scientific, hep-ph/0201005; Yu.A.Simonov and J.A.Tjon, Ann.Phys. 300, (2002) 54; hep-ph/0205165

[25] M.A.Shifman, A.I.Vainshtein and V.I.Zakharov, Nucl. Phys.B147, 385 (1979)

[26] V.I.Shevchenko and Yu.A.Simonov, Phys. Rev.D65 (2002)074029; hep-ph/0109051

[27] Yu.A.Simonov, Nucl. Phys. B324 (1989) 67

[28] W.Lucha, F.Schöberl and D.Gromes, Phys. Rept. 200 (1991) 127

[29] M.Eidemueller, M.Jamin, Phys. Lett. B416 (1998) 415, hep-ph/9709419,

V.I.Shevchenko, hep-ph/9802274

[30] V.I.Shevchenko and Yu.A.Simonov, Phys. Lett. B437 (1998) 131, hep-ph/9803377.

[31] V.I.Shevchenko and Yu.A.Simonov, Phys. At. Nucl. 60 (1997)1201.

[32] M.Goeckeler et al.(QCDSF-UKQCD Collaboration), hep-lat/0409166 
[33] Yu.A.Simonov, Sov. J. Nucl. Phys. 3 (1966) 461; A.M.Badalian and Yu.A.Simonov, Sov. J. Nucl. Phys. 3 (1966) 755; 5 (1967) 60; F.Calogero and Yu.A.Simonov, Phys. Rev. 169 (1968) 789

[34] Yu.A.Simonov, Phys. At. Nucl. 66 (2003) 2033; hep-ph/0212253

[35] Yu.A.Simonov, hep-ph/0406290

[36] A.Yu.Dubin, A.B.Kaidalov, Yu.A.Simonov, Phys. Atom. Nucl. 56, (1993) 1745, hep-ph/9311344

[37] Yu.A.Simonov, Phys. Atom. Nucl. 64, 1876 (2001), hep-ph/0110033

[38] Yu.A.Simonov, Phys. At. Nucl. 64, 548 (2001)

[39] Yu.A.Simonov, hep-ph/0407027 